\title{
Adhesion of a Living Hyaline Cartilage Graft as an Industrial Product for Cartilage Repair
}

\begin{abstract}
Nie Xiaolei
Nanyang Technological University School of Chemical and Biomedical Engineering Bioengineering Department phD student

Wang Dongan

Nanyang Technological University School of Chemical and Biomedical Engineering Bioengineering Department Associate Professor

Abstract: Living hyaline cartilage graft (LhCG) is a scaffold free $3 D$ pure hyaline type cartilage engineered graft. It is fabricated based on the phase transfer cell culture method of chondrocytes in a multi-cavity scaffold build up on the mixture of alginate and gelatin microspheres. This graft has very good animal test result in terms of cartilage damage repair. However due to the source of the primary chondrocytes that are adopted in the fabrication of the graft, there is large batch to batch variance in the quality of the graft. Therefore in order to suit for the industrial purpose, the graft is adhered by bio-glue before the decellularization process. Genipin as a popular bio-glue is tested out in the work reported in this paper to figure out the best concentration generally appropriate for the graft. A concentration gradient of $2 x$ from $0.05 \%$ to $0.4 \%$ are applied to the graft and compared with the control group of PBS. It is found that $0.05 \%$ genipin is too low to ensure the integrity of the graft throughout the harsh decellularization process, while the $0.2 \%$ and $0.4 \%$ concentrations are too high to allow the decellularization process. Therefore it is concluded in this paper that $0.1 \%$ concentration of genipin is the optimized concentration. In the future, to make sure the quality of the graft, chondrocytes quality control measurement are to be figured out. Otherwise, other tissue glues, other than genipin could be assayed to verify the effect of gelation for the LhCG graft.
\end{abstract}

Keywords: Biomedical engineering, Regeneration engineering, Tissue engineering

\section{INTRODUCTION}

The knee injury is a main reason that ends a sportsmen's career [1]. Currently, there are several methods that is treating the knee injuries. They are autoplastic surgery, autologous chondrocytes implantation (ACI) and membrane autologous chondrocytes implantation (mACI) [2]. Among all these methods, autoplastic surgery is actually a palliative care approach, while ACI and mACI are in fact tissue engineering method without any scaffolds. However these treatments are facing low effectiveness in terms of repair. A tissue engineering method is more effective since the scaffold provides a habitat for the cells to reside and proliferation.

Previously mentioned methods are currently applied clinically is due to its low toxicity. The scaffold in traditional tissue engineering methods is the main source of the toxics. The scaffolds are normally hydrogel, either natural or synthetic. The naturally derived hydrogels are biocompatible, but it may induce immune response. The synthetic hydrogel could be toxic or its degradation produce could be toxic. Therefore a scaffold free graft is in great demand to close the gap between the clinical application reality and laboratory theory. The lab where the author is in has developed a scaffold free cartilage graft for the repair of knee damage and gained promising results until the level of large animal models, mini pig. However, an autologous living graft has many challenges when it is moved up into the industrialization process.

The graft is facing an expiration period issue, due to the culture progress of the graft and the storage difficulty of a living piece of tissue. Therefore decellularization is the next step in mind to prepare the graft for industrialization. Decellularization not only resolves the issue of fast degeneration of the graft after maturation, but also it enlarges the source of cells in growing the graft. It is because after the removal of chondrocytes, the graft is an acellular implant, the ligands and recognition molecules inserted on the membrane surface of the cells are removed as well. The graft now is an immune 
response free implantation.

In the decellularization process, it is found that the final status of the graft has variations from batch to batch due to the inert variations on cells status that were used in graft fabrication. Therefore it is proposed that the grafts are to be gelled up by biological glues before the decellularization process to achieve a standard quality of final graft. The biological glue selected is genipin, which is a FDA approved biological glue to help seal the sutures in surgery. Different concentration of the glue is applied to the graft to test the most suitable concentration for the graft.

\section{MATerials AND Methods}

\subsection{Fabrication of Scaffold Free Cartilage Graft}

The chondrocytes are harvested from the porcine hind leg femur distal end cartilage. The cells are cultured on T175 flask to proliferation until $90 \%$ confluence. The culture medium contains $84 \%$ DMEM, $15 \%$ serum and $1 \%$ penicillin and streptomycin.

After the proliferation, the cells are digested and mixed together with gelatin microspheres in alginate sodium solution. The concentration of cells are $10^{7}$ cells $/ \mathrm{ml}$. The concentration of the gelatin microsphere is $0.3 \mathrm{~g} / \mathrm{ml}$. When calcium chloride ions are added into the solution system. The calcium ions crosslinked the alginate to generate a fast phase transformation from solution to gelation within four minutes. Under the culture condition of $37{ }^{\circ} \mathrm{C}$ and $5 \% \mathrm{CO}_{2}$ the gelation microspheres dissolve in several days' time. As a result, a multi-cavity structure is formed. The chondrocytes are able to grow into the cavities left out after the dissolution of the gelation microspheres. This is patented by the lab "phase transfer cell culture".

This type of three dimensional culture of chondrocytes in alginate significantly increase the total cell number after a period of 35 days. After the maturation of the graft. The alginate is removed by immersing the whole graft in sodium citrate $(55 \mathrm{mM})$ for 10 mins. Finally the graft is culture for another 10 days to let the cell to further proliferate in the space that is left out after the removal of alginate. In the whole three dimensional culture process, the graft is in a medium consists of 76\% DMEM, 20\% serum, $1 \%$ non-essential amino acid, $1 \%$ HEPES buffer, $1 \%$ penicillin and streptomycin. $0.5 \%$ Vitamin $\mathrm{C}$ and $0.4 \%$ L-proline [3].

\subsection{Decellularization}

Hypotonic solution, Triton-X and DNase RNase solution are utilized to remove cells from the graft. When immersed in the solutions, the graft shakes to ensure the effectiveness of the decellularization process.

\subsection{Gelation of Graft Before Decellularization}

Genipin is used with a concentration gradient of $0.05 \%(\mathrm{w} / \mathrm{v}), 0.1 \%, 0.2 \%$ and $0.4 \%$. The genipin is dissolved in $70 \%$ ethanol (analytical grade). The graft after the totally 45 days of living three dimensional culture is immersed in the genipin solution for 48 hours. The control group is immersed in phosphor buffer saline for 48 hours instead. During this 48 hours, the graft is maintained under the $37{ }^{\circ} \mathrm{C}$ and $5 \% \mathrm{CO}_{2}$ condition.

The macroscopic appearance is observed after the gelling up and after the decellularizing of the graft. (Graph 1) to generally check the integration of the whole graft after the gelling up and decellularization.

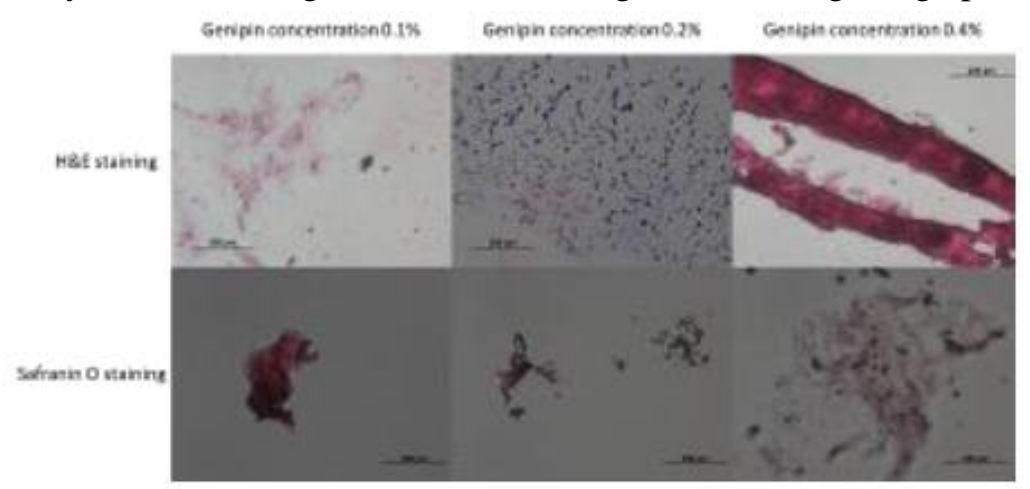

Figure2. Histology of the Decellularized Gelled Graft. the First Row is the Staining of H\&E, While the Second Row is the Staining of Safranin O. From First Column to Third Column the Concentration of Genipin Applied Increases.

The decellularized graft underwent histology studies to show the effectiveness of the decellularizaion. 


\subsection{Histology}

After gelation the graft is first observed under microscope for the general appearance after the gelling up and decellularization.

In order to observe the character of the graft, histological staining are conducted. H\&E, Safranin O and immunochemical staining of Collagen II are used to verify the quantity of cells, extracellular matrix, glycosaminoglycan and type II collagen.

LhCG constructs after treatments were fixed with $3.7 \%$ formaldehyde overnight. The formaldehyde is replace by $30 \%$ sucrose in PBS solution (w/v) and then the medium is changed to sucrose solution and tissue freezing medium (OCT, Leica Biosystems) in a ratio of 1:1. Finally, all the medium is changed to tissue freezing medium. The samples were then cryo-sectioned and subject to hematoxylin and eosin (H\&E) staining for histological observation using standard protocol. Safranin O staining (sigma, USA) was performed to evaluate glycosaminoglycan (GAG) deposition in the extracellular matrix (ECM) according to manufacturer's protocol. DAPI staining was performed to observe the cell nucleus.

Immunohisto chemistry staining of collagen type II was also performed. The sections were blocked with $10 \%$ horse serum at $37^{\circ} \mathrm{C}$ for 20 mins. After washed with PBS, the slides were incubated respectively with polyclonal rabbit IgG anti-collagen type II (Santa Cruz) at $4^{\circ} \mathrm{C}$ overnight. Subsequently, the slides were washed with PBS and incubated with goat anti-rabbit IgG-FITC (Santa Cruz) at $37^{\circ} \mathrm{C}$ for 1 hour. The nuclei were then counterstained by DAPI and observed under fluorescence microscopy.

\section{Results}

After the decellularization only the concentrations of $0.1 \%, 0.2 \%$ and $0.4 \%$ is able to maintain the integrity of the entire graft. They are directly observed under optical microscope. The microscopic images are arranged side by side following an increasing concentration order from $0.1 \%$ to $0.4 \%$ in a double gradient.
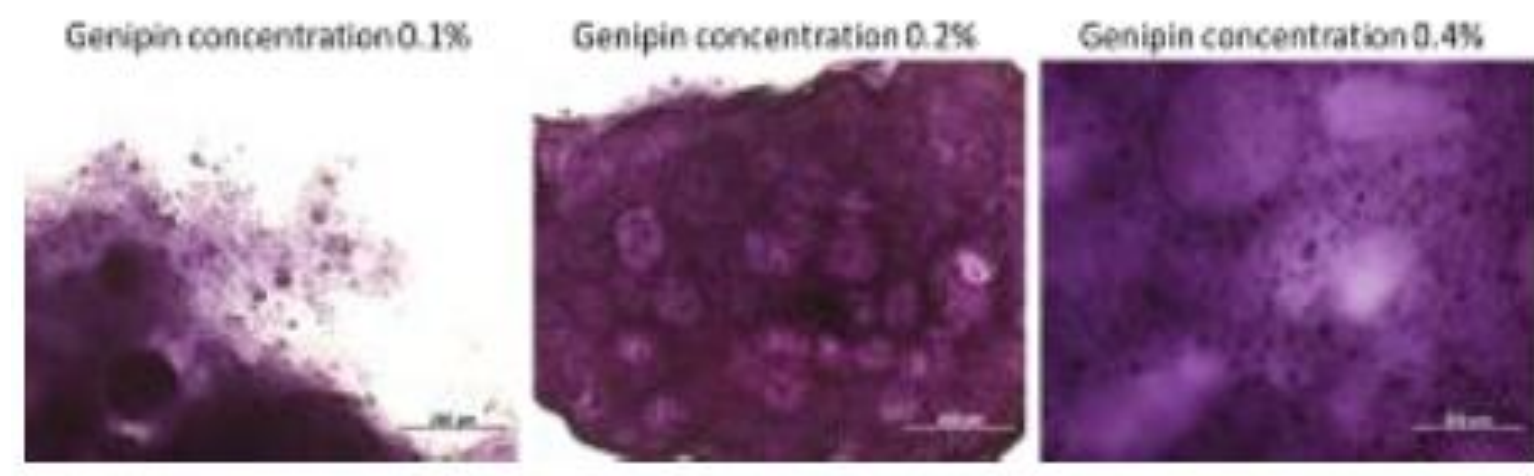

Figure1. The observation under optical microscope of the three decellularized gelled LhCG with different concentrations of the genipin applied to the graft.

It is observed that after the addition of genipin onto the graft. The graft is turned into blue. It is because of the gelation of collagen by genipin. It results in the color change.

To verify the effect of decellularization process in the condition of gelation histology is conducted to qualitatively manifest the collagen, glycosaminoglycan and cell abundance of the graft.

The H\&E staining is able to stain the nucleus and the extracellular matrix. Safranin $\mathrm{O}$ is able to stain the glycosaminoglycan, which is a main component in cartilage.

The immunochemical histology is conducted to test the Collagen II left after the decellularization.

From the microscopic observation it is conclusive that all the three concentrations of genipin are able to connect the tissues together and make the tissue able to undertake the relative harsh decellularized condition. 


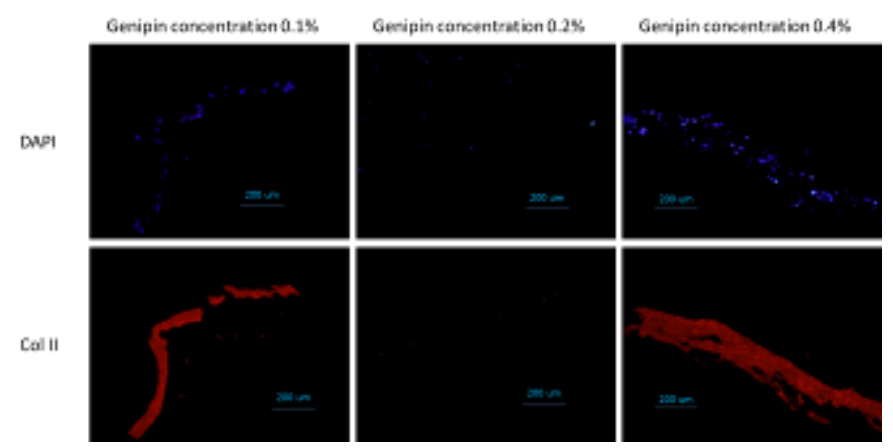

Figure3. Immunochemical histology of the decellularized gelled graft. The pictures in the same column is taken from the same position. Meanwhile each column is a different genipin concentration. The concentration increases from left to right with concentration $0.1 \%, 0.2 \%$ and $0.4 \%$. The first row is the DAPI staining for the nuclei. The second row is the stain for Collagen II.

Moreover, from the histology it is observable that $0.1 \%$ and $0.2 \%$ retains relatively larger amount of extracellular matrix (ECM) compared with the $0.4 \%$. While the $0.4 \%$ retains the largest amount of glycosaminoglycan. It implies that although the glycosaminoglycan is less in lower concentration of genipin, the collagen as another major component of the ECM are more conserved in lower concentration.

This thought is further confirmed by the immunochemical histology. The Collagen II staining normalized to DAPI staining is the most abundant in the $0.1 \%$ concentration group. It can interpreted that although the collagen II staining is high but the DAPI staining is also high. However in the $0.1 \%$ group, the collagen II staining color is roughly the same as the $0.4 \%$ group, while the DAPI staining is much less than the $0.4 \%$ group. The decellularization process aims to achieve a cell free and ECM abundant graft. Therefore the higher the ratio of collagen II to nuclei, the better the graft. As a result it is concluded that $0.1 \%$ concentration of genip in is the best concentration.

\section{DisCuSSION}

The genipin is an extraction from gardenia fruits [4]. It is widely used in regenerative medicine mostly as a tissue adhesive. In this research work. The glue is used to gel up the Living hyaline Cartilage Graft (LhCG). LhCG is developed using the native or primary chondrocytes harvested directly from the pig hind limbs. Therefore the quality is highly dependent on the age or health status of the pig. Hence, there is very large batch to batch variance.

From the microscopic observation it is clear that from $0.1 \%$ to $0.4 \%$ all are able to maintain the integrity, while the $0.05 \%$ concentration is not able to stand the harsh decellularization process. The reason why the picture is blue is because that after the crosslinking by genipin, the color of collagen is turned blue. The blue color is due to the oxidization of Lysine and Hydroxylysine. [5]

In the histology test, $H \& E$ stains for the nuclei and extracellular matrix (ECM) respectively. While the Safranin O stains for the glycosaminoglycan (GAG). It is shown that in the $0.1 \%$ concentration, the stain for the ECM is the strongest while the stain for the nuclei is the least. However, in a higher concentration group, for example, in $0.2 \%$ group, the stain for nuclei is already quite strong. While for the $0.4 \%$ group, the stain is too strong to differentiate nuclei from the ECM. In the stain for GAG, $0.4 \%$ groups shows the strongest stain, and the lower concentrations' stain could be a fake positive stain.

In the immunohistology, Collagen II is detected and at the same time a DAPI counterstain for the nuclei. It is obvious that both $0.1 \%$ and $0.4 \%$ has high presence of the fluorescence for Collagen II. However the $0.4 \%$ group illustrated strong stain for nuclei too.

\section{Evaluation}

In the microscopic observation, it is not easy to observe the structure of the graft. It is mainly because of the thickness of the graft. Therefore the graft needs to be sectioned to have a detailed observation.

From the histological and immunohistological chemistry staining, it is understandable that the H\&E staining and Collagen II staining confirms each other about the best concentration of the genipin. Among the three tested groups, $0.1 \%$ is the best concentration. A lower concentration of $0.05 \%$ is not 
able to maintain the integrity. Therefore it is possible that the optimized concentration is in between $0.005 \%$ to $0.1 \%$. This could be further simplified and currently the best concentration is $0.1 \%$.

The Safranin O staining is low in the $0.1 \%$ but is high in the $0.4 \%$. This could be due to the gelation mechanism of genipin. Genipin mainly crosslinked the lysine and hydroxylysine. These amino acids are abundant in collagen but is not so abundant in GAG. However in a higher concentration, it is possible that the oxidization by radical reactions are able to react with GAGs too. Therefore the GAG is more conserved in the $0.4 \%$.

The immunohistological chemistry staining is clearly illustrating that the higher concentration is able to retain the Collagen II, however the nuclei is not removed too. In the $0.2 \%$ group, both the nuclei and Collagen II are very weak, whereas in the $0.4 \%$ both the nuclei and Collagen II are both very strong. Therefore it suggests that genipin is able to link up the proteins but simultaneously, the cells are wrapped and kept with the collagen network. Therefor it seems to be implying that although the gelation by genipin is able to maintain the integrity of the graft but at the same moment, it makes the decellularization process more difficult. Therefore it shows the importance of the original property of the graft.

\section{Conclusion}

The result is showing the simultaneity of the existence of the ECM and the nuclei. It is suggesting the important of the original quality of the graft. Therefore in order to make the quality of the graft more stable to suit for the industrial purpose, the quality of the chondrocytes are to be controlled. If the gelation is finally still need to be conducted to ensure the integrity of the graft after decellularization, other tissue adhesions other than genipin could be considered.

\section{ACKNOWLEDGMENT}

X. Nie thanks Dr. Wang Dongan for his help in this research.

\section{REFERENCES}

[1] Ristolainen, L, Kettunen, JA, Kujala, UM, \& Heinonen, A, "Sport injuries as the main cause of sport career termination among Finnish top-level athletes", European Journal of Sport Science, vol. 12, no. 3, pp. 274-282, May 2012

[2] Bartlett, W, Gooding, CR, Carrington, RJ, Skinner, JA, Briggs, TR, \& Bentley, G, 'Autologous chondrocyte implantation at the knee using a bilayer collagen membrane with bone graft. A preliminary report', The Journal of Bone and Joint Surgery. British Volume, vol. 87, no. 3, pp. 330-332, Mar. 2005

[3] Su, K, Lau, T, Leong, W, Gong, Y, \& Wang, D 'Creating a Living Hyaline Cartilage Graft Free from Non-Cartilaginous Constituents: An Intermediate Role of a Biomaterial Scaffold', ADVANCED FUNCTIONAL MATERIALS, vol. 22, no. 5, pp. 972-978, Mar 2012

[4] Muzzarelli, RA 2009, 'Review: Genipin-crosslinked chitosan hydrogels as biomedical and pharmaceutical aids', Carbohydrate Polymers, vol. 77, pp. 1-9, Jan. 2009

[5] R. Touyama, K. Inoue, Y. Takeda et al., "Studies on the blue pigments produced from genipin and methylamine. II. On the formation mechanisms of brownish-red intermediates leading to the blue pigment formation," Chemical and Pharmaceutical Bulletin, vol. 42, no. 8, pp. 1571-1578, Jan. 1994.

\section{AUTHOR'S BIOGRAPHY}

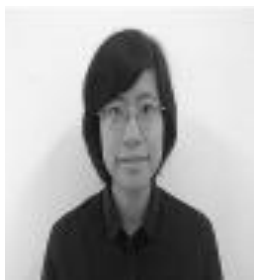

Nie Xiaolei, received the B.Eng. degree in Bioengineering from Nanyang Technological University, Singapore in 2014. She is currently pursuing the Ph.D. degree in Chemical and Biomedical Engineering at Nanyang Technological University, Singapore.

In 2013, she was an Intern at Johnson \& Johnsons, Singapore. From 2014 to 2015, she was an Operation Manager in H\&Y TCM clinic Pte. Ltd., Singapore. Her research interest includes anti-cancer drug development and tissue engineering. 